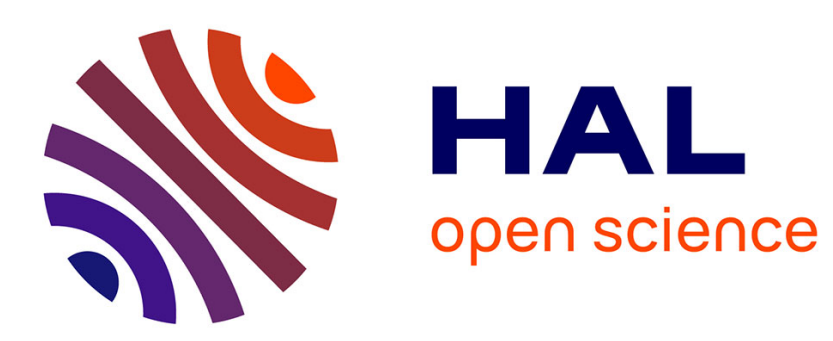

\title{
Règlement INCO : application en restauration collective; exemple des collèges du Doubs
}

\author{
E. Capelli, J.-M. Rame, A. Doc
}

\section{To cite this version:}

E. Capelli, J.-M. Rame, A. Doc. Règlement INCO : application en restauration collective; exemple des collèges du Doubs. Revue francaise d'allergologie, 2019, 59, pp.196 - 198. 10.1016/j.reval.2019.02.194 . hal-03486370

\section{HAL Id: hal-03486370 \\ https://hal.science/hal-03486370}

Submitted on 20 Dec 2021

HAL is a multi-disciplinary open access archive for the deposit and dissemination of scientific research documents, whether they are published or not. The documents may come from teaching and research institutions in France or abroad, or from public or private research centers.
L'archive ouverte pluridisciplinaire HAL, est destinée au dépôt et à la diffusion de documents scientifiques de niveau recherche, publiés ou non, émanant des établissements d'enseignement et de recherche français ou étrangers, des laboratoires publics ou privés.

\section{다)(1) $(5$}

Distributed under a Creative Commons Attribution - NonCommercial| 4.0 International 


\section{Règlement INCO : Application en restauration collective ; Exemple des collèges du Doubs}

Application of the INCO regulation in mass catering; the example of secondary schools in the Doubs region of France

E. Capelli a, *, d - J.-M. Rame b,c - A. Doc b,c,d

\footnotetext{
a, Département du Doubs, 7 avenue de la Gare d'eau, 25031 Besançon cedex, France -03 81258497 - emilie.capelli@doubs.fr $b$ Réseau d'Allergologie de Franche-Comté, 2, place Saint-Jacques, Arsenal, 25030 Besançon cedex, France

c, CHU J.-Minjoz, boulevard Fleming, 25030 Besançon cedex, France

d, Allergodiet, Groupe de Travail de la Société Française d'Allergologie (SFA)
} 


\title{
Résumé
}

Une collaboration entre la diététicienne du Département du Doubs et le RAFT (Réseau d'Allergologie de Franche Comté), a permis de former tous les personnels des collèges du Doubs à l'accueil des élèves allergiques alimentaires dans les restaurations scolaires, ainsi qu'à l'affichage des allergènes conformément au règlement INCO. Plusieurs enquêtes menées auprès des participants ont mis en lumière une forte satisfaction vis-à-vis de cette formation, ainsi qu'un taux d'affichage des allergènes de $100 \%$ dans les collèges, deux ans après les sessions.

Mots-clés: Allergie Alimentaire, Restauration Collective, Diététicien Nutritionniste, Formation

\begin{abstract}
A collaboration between the dietician of the Department of Doubs and the RAFT (Franche Comté Allergology Network), has made it possible to train all the staff of Doubs colleges to the reception of allergic food students in schools restorations, as well as the display of allergens according to the INCO regulation. Several surveys conducted of participants revealed a high level of satisfaction with this training, as well as a $100 \%$ allergen display rate in colleges, two years after the sessions.
\end{abstract}

Keywords: food allergy, mass catering, dietician, training

En milieu scolaire, la mise en place du Projet d'Accueil Individualisé (PAI) de façon de plus en plus systématique a contribué à l'amélioration de l'accueil des enfants présentant une allergie alimentaire (AA) en restauration collective (RC). Cependant, ils sont encore parfois confrontés à deux difficultés : des réticences d'accueil ou à l'inverse une négation du risque allergique par certains personnels, pouvant mettre en danger l'enfant. Ces attitudes inadaptées sont liées à la méconnaissance de l'AA et des bonnes pratiques d'accueil des enfants concernés, par des personnels de collectivités ne bénéficiant que trop rarement, voire jamais de formation dans ce domaine.

\section{$\underline{\text { Affichage des allergènes en RC }}$}

En 2011, la publication du règlement Européen INCO (INformation COnsommateur), a instauré de nouvelles règles concernant l'obligation d'affichage des 14 allergènes à déclaration obligatoire (ADO). Ce règlement a modifié les obligations en matière d'information des consommateurs sur les denrées alimentaires, il prévoit notamment "l'obligation d'informer le consommateur sur la présence de tout ingrédient ou auxiliaire technologique ou dérivé d'une substance ou d'un produit provoquant des allergies ou intolérances listées en annexe II du règlement INCO ». Cette annexe liste les 14 ADO. 
Pour les denrées non pré-emballées, chaque Etat membre a dû préciser les modalités d'application : en France, le décret 2015-447 du 17 avril 2015 [1] a donc été publié. Les RC sont concernées par cet affichage, et le règlement précise que "L'information mentionnée à l'article R. 112-11 est indiquée sur la denrée elle-même ou à proximité de celle-ci de façon qu'il n'existe aucune incertitude quant à la denrée à laquelle elle se rapporte »

Formation des personnels de RC dans le Doubs

\section{Méthode}

Le Département du Doubs (CD25) a créé un poste de diététicien-nutritionniste (DN) en 2012 dédié à la $\mathrm{RC}$ des 46 collèges du territoire. Elle a repéré à son arrivée un besoin de formation des agents des collèges sur le thème des AA et a contacté le RAFT (Réseau d'Allergologie de Franche Comté).

Le RAFT a conçu et animé des formations grâce à sa DN et son médecin coordonnateur (MC) dans le cadre du Plan Régional Santé Environnement 3. Ces formations étaient gratuites, car le RAFT, UF du CHU de Besançon, est financé par l'ARS dans le cadre de l'enveloppe du Fonds d'intervention régional. Ainsi, dès l'année scolaire 2013-2014, la DN du CD25 a pu élaborer un plan de formation à destination des agents de restauration, des adjoints gestionnaires et des infirmières scolaires, en y associant les médecins scolaires des secteurs concernés.

Suite à la publication du règlement INCO en 2015 [1], une nouvelle collaboration entre la DN du CD25 et le RAFT s'est mise en place afin de proposer une deuxième session de formations en y intégrant les nouveautés réglementaires.

La formation de 2013-2014, d'une durée de 2h30, était composée de deux diaporamas interactifs. Le premier, présenté par le MC du RAFT, concernait les aspects médicaux de l'AA, puis la DN présentait les aspects hygiéno-diététiques de l'AA. Deux ateliers pratiques en alternance étaient proposés à tous les participants, animés par le MC et la DN du RAFT, respectivement sur "la présentation du PAI et la trousse d'urgence " avec manipulation des stylos auto-injectables d'adrénaline, et sur « la lecture des étiquettes ».

La formation de 2016-2017 a repris le même format : diaporamas, puis ateliers pratiques. La lecture des étiquettes se faisait cette fois dans le cadre de l'affichage INCO. Deux documents ont été créés suite à ces sessions : une affiche récapitulant les caractéristiques des 14 ADO pour les cuisiniers, et une brochure de conseils pratiques « Comment accueillir un enfant AA au collège ? ".

\section{Résultats des formations:}

Les deux sessions ont permis de former respectivement 127 et 112 personnes, soit $87 \%$ de cuisiniers convoqués, $70 \%$ des adjoints gestionnaires, et $57 \%$ des infirmières. $100 \%$ des médecins de secteurs s'étaient également déplacés. 
Un questionnaire a été adressé à tous les participants de la première session en 2016, soit 2 ans après la première formation. Les résultats ont mis en lumière une grande satisfaction des participants sur tous les points interrogés et ils ont fait l'objet d'une publication dans la Revue Française d'Allergologie [2].

Suite à la deuxième session qui intégrait INCO (en 2016-2017), un nouveau questionnaire a été distribué aux cuisiniers juste après la formation, afin d'évaluer le taux d'affichage règlementaire. Début 2019, soit deux années après cette évaluation, un troisième questionnaire a été adressé aux 46 collèges, afin d'évaluer la persistance de l'affichage à deux ans.

Lors du deuxième questionnaire post-formation de 2016-2017, 56\% des cuisiniers ont répondu. 96,3\% d'entre eux ont trouvé cette formation utile pour l'affichage des 14 ADO. $40 \%$ ont déclaré avoir mis en place l'affichage avant la formation, contre $79 \%$ après la session. Cependant, seuls $32 \%$ des répondants déclaraient faire cet affichage sans difficulté. Les principaux freins déclarés étaient : pour $50 \%$ un manque de temps et pour $32 \%$ un excès de tâches administratives (Fig. 1).

Lors du troisième questionnaire envoyé en $2019,46 \%$ des cuisiniers ont répondu. $85 \%$ ont trouvé les formations utiles, $100 \%$ ont déclaré avoir mis en place l'affichage INCO, et $75 \%$ ont indiqué n'avoir aucune difficulté à le mettre en place. Le manque de temps n'est plus cité qu'à $10 \%$, et l'excès de tâches administratives qu'à 15\% (Fig. 1). 35\% déclarent y consacrer moins de 5 min par jour, 60\% y consacrent 5 à $10 \mathrm{~min}$, et seuls $5 \%$ y passent 10 à $15 \mathrm{~min}$.

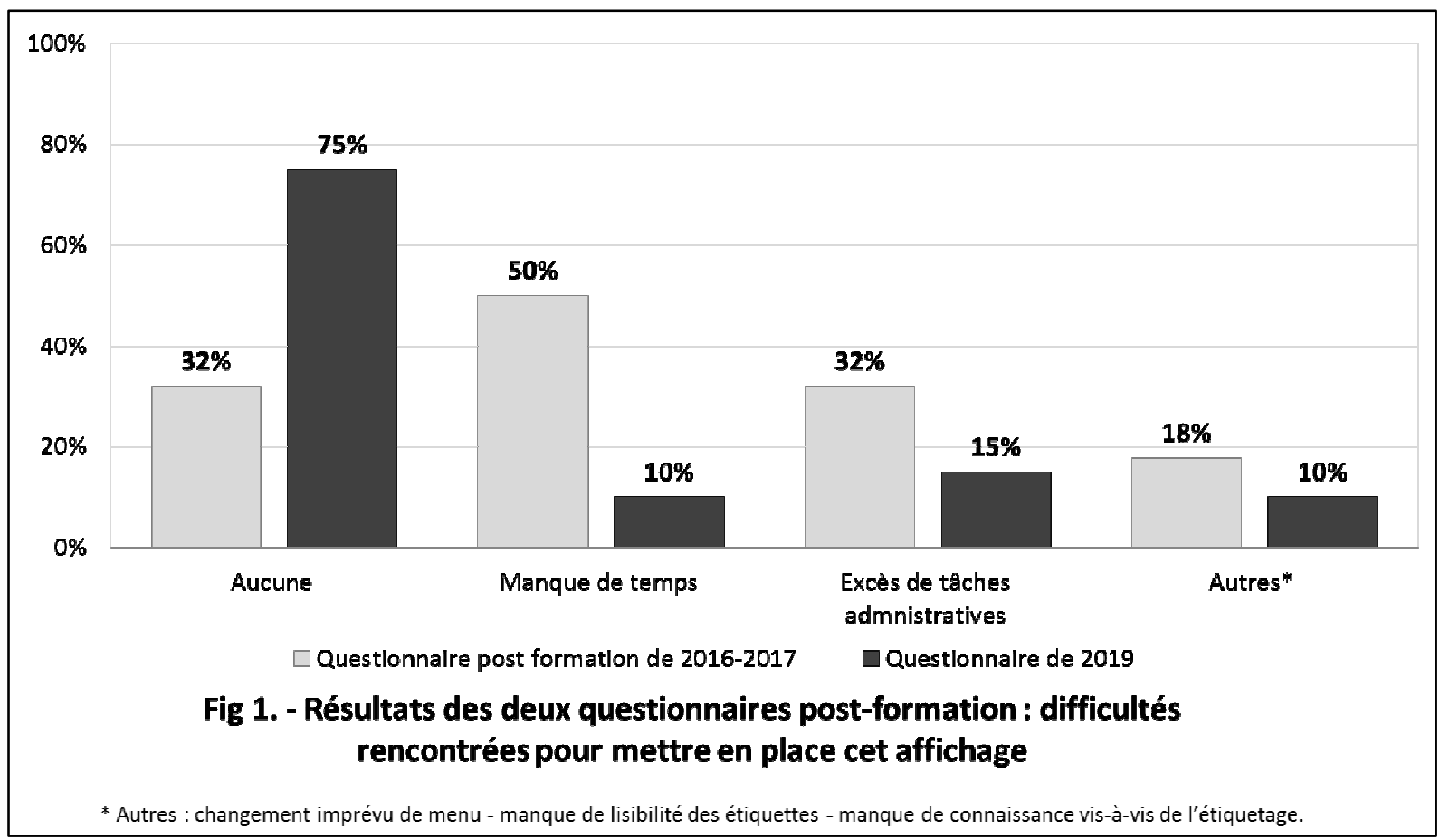

3. Discussion : 
Le deuxième questionnaire a démontré que l'affichage des 14 ADO a doublé juste après la formation de 2016-2017, passant de 38\% à 79\% l'affichage. Les taux de satisfaction vis-à-vis des sessions sont restés élevés. Lors de cette même enquête, $32 \%$ seulement des répondants déclaraient n'avoir aucune difficulté à mettre en place cet affichage, contre $75 \%$ en 2019. Avec le temps, cette tâche est perçue comme moins compliquée et elle prend peu de temps. II est également important de souligner qu'après 2 ans de recul, la totalité des établissements applique les règles d'affichage INCO.

La réussite de cette action menée dans le Doubs, s'explique par plusieurs facteurs :

- Tous les professionnels du collège concernés par l'accueil de l'élève AA ont suivi ensemble la même formation. Ils ont pu échanger sur leurs pratiques, leurs craintes comme leurs compétences respectives.

- Les formations ont été dispensées par des professionnels expérimentés dans l'AA en milieu scolaire, qui ont adapté leurs présentations (vocabulaire approprié, mises en situations pratiques).

- La DN du CD25 est en contact avec les collèges régulièrement et apporte des réponses aux questions ou problèmes rencontrés directement sur place, instaurant ainsi une continuité avec les formations dispensées.

S'il a été possible d'évaluer le taux d'application de l'affichage INCO dans les collèges du Doubs, il est plus compliqué d'en évaluer le contenu. En effet, la DN du CD25 se rend régulièrement sur site et a pu constater que les erreurs d'affichages, même si elles ne sont pas fréquentes, existent. Les erreurs relevées sont en général dues à des oublis : soit des oublis de plats présentés qui proviennent de surplus de la veille, soit d'ingrédients bruts sans étiquettes. Par exemple, dans un céleri rémoulade, le cuisinier va noter l'œuf et la moutarde de la mayonnaise car il a lu l'étiquette et reporté les allergènes, mais va omettre le céleri qu’il a épluché et râpé.

Les agents de restauration sont des agents départementaux, ils ont donc été convoqués par le Département. Les adjoints gestionnaires comme les infirmières scolaires, sont des agents $d^{\prime} E t a t$ : ils ont été invités à participer, seulement s'ils le souhaitaient. Malgré ce caractère "facultatif ", le pourcentage de participants a été élevé, ce qui reflète un réel intérêt pour le sujet de la part de ces professionnels.

\section{Conclusion:}

La Société française d'allergologie a émis des propositions en 2016 concernant les conditions d'établissement du PAI. Ces recommandations soulignent tout l'intérêt de l'information de l'entourage du patient dans le milieu scolaire [3]. Les formations que nous avons proposées s'inscrivent donc pleinement dans ces recommandations. Les résultats très positifs des enquêtes menées auprès des participants démontrent un réel besoin de formation dans le 
domaine des AA des agents des collèges, qui, avec les outils adéquats, sont également moins démunis face aux obligations règlementaires en termes d'affichage des 14 ADO.

Conflit d'intérêt : aucun.

\section{Références}

[1] Règlement (UE) n o 1169/2011 du Parlement Européen et du Conseil du 25 octobre 2011 concernant l'information du consommateur sur les denrées alimentaires et notamment ses articles 9, 21, 44 et l'annexe II sur les substances ou produits provoquant des allergies ou intolérances. Décret no 2015-447 du 17 avril 2015 relatif à l'information des consommateurs sur les allergènes et les denrées alimentaires non préemballées.

[2] J -M. Rame, A. Doc, J. Raibaut, E. Lalaurie. Allergie alimentaire (AA) et restauration collective : comment améliorer l'accueil des élèves ? Bilan de l'expérience menée en Franche-Comté. Rev Fr Allergol 2017 ; 57; 67-76.

[3] Pouessel G, Deschildre A, Beaudouin E, Birnbaum J, Neukirch C, MeiningerC, Leroy S. Conditions d'établissement du projet d'accueil individualisé pour l'enfant allergique : position des groupes de travail " anaphylaxie ", " allergie alimentaire " et "insectes piqueurs » sous l'égide de la Société française d'allergologie. Rev Fr Allergol 2016;56:434-40. 